\title{
WEB LAND - DESIGN TRENDS FOR 2016
}

\author{
Luminiţa GIURGIU, Ghiţă BÂRSAN \\ "Nicolae Bălcescu" Land Forces Academy, Sibiu, Romania \\ lumigee@armyacademy.ro, gbarsan@armyacademy.ro
}

\begin{abstract}
The reason for building a website is primarily to reach target audiences worldwide (or local audiences, as appropriate) and, secondly, to represent a company or organization in the online environment. And if we take the second case, namely online representation is necessary to make sure that website is always updated and that has an improved web design. Trends in web design can have multiple roles: to make a website more attractive and more functional and to increase its traffic and subsequent conversions. Given the evolution of technology, most websites have to suffer some changes so frequent visitors to enjoy right experiences regardless of how they access, so for companies that take seriously the importance of their online website, respecting future trends in the online space is the only way to start a new project. This article brings up some trends in web design, considering this issue of great interest in the online environment. Although it is quite difficult to predict which trends will later develop into the necessity, we must keep pace in this digital world where everything seems to change so fast.
\end{abstract}

Keywords: web, design, trends

\section{Responsive Web Design}

Responsive Web Design is brought into discussion even it would be pretentious to call it a trend because it has become rather a necessity, see figure 1 . A website that is not responsive will need a special version for mobile devices to be displayed by search engines after searches conducted by users of smart phones or of the tablets, not to mention watches or Smart TVs. Design flexibility is thus a requirement of the market before it constitutes an advantage over competitors.

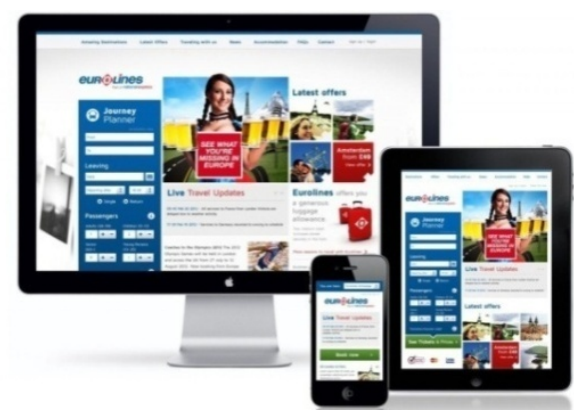

Figure 1 Responsive Design
A good result on the search results pages of search engines will be obtained if the site features a responsive web design because Google will index the pages easier recommending them to the interested users.

Yet a small observation: the responsive design that manages pages for both PCs or laptops and mobile phones, tablets and so on, can lead to the realization of pages that is not as expressive for both types of devices. Although this topic stirs controversy about the effort to achieve pages responsiveness, the movement that promotes this trend, launched since 2015, demand the right to equality in design and navigation, whether a site is accessed from mobile, tablet, PC or, soon, the Smart Watch sites.

\section{Shaped Hamburger Menu}

This trend refers to a simplified navigation hidden in a menu hamburger type. There is a tendency included also in 
desktop versions of sites. We are already accustomed when accessing websites on mobile devices to see a specific icon called hamburger. But this simplified navigation way for mobile devices has entered desktop variants in some sites, being used for the main menu and in others to a secondary navigation menu. Important sites such as Time or producing software house Adobe already uses it as a main navigation menu, see figure 2, and others like that of $\mathrm{CNN}$ uses this typology to a secondary menu. This is a trend that is gaining ground especially for websites that store a lot of information that should be optimized for navigation space. In addition this technique offers a minimalist site appearance that constitutes another trend already noticed and constantly growing.

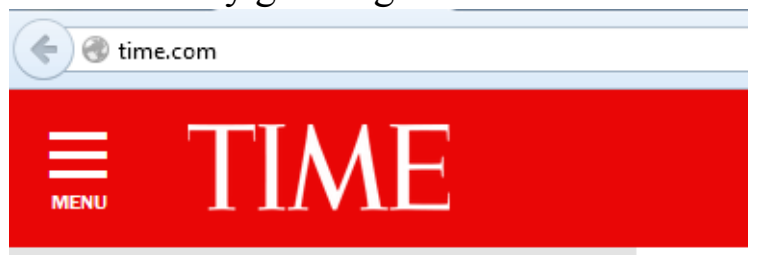

\section{Vertical Pages and Scroll}

In other words - Site One Page it seems that sites with many pages were abandoned this year. It all started with the iPhone6 page, which demonstrated that it is possible to enter a lot of information on one page in an elegant and convincing manner. With the explosion in the number of smart phones users, the trend is that almost all sites to be structured lengthwise. Regardless of the new techniques adopted by web designers, the option of scrolling is and will continue to be one of the most used because, in close liaison with the responsive design, this is highly requested and appreciated by mobile device users. Scrolling considerably reduces additional loading page time and simplifies navigation by providing a fluency in completing the information on a website. Thus, easy and enjoyable navigation entails new visitors. On a site with this type of design it is much easier to navigate compared to the classic type design with many pages involving several clicks. Another advantage is that the new design is SEO friendly.

\section{Parallax Scrolling}

When he emerged, this effect has produced a real revolution. Parallax scrolling creates a 3D effect during scrolling web page by moving background in a slower pace compared to the foreground, see figure 3. CSS3 and HTML5 together have made possible the multi-layer scrolling. In this web design wallpaper tends to move more slowly than the rest of the site content. The result is a $3 \mathrm{D}$ effect that not only looks great, but adds depth to a page. This effect is used both for backgrounds with images and for those with videos, and is certainly a trend that

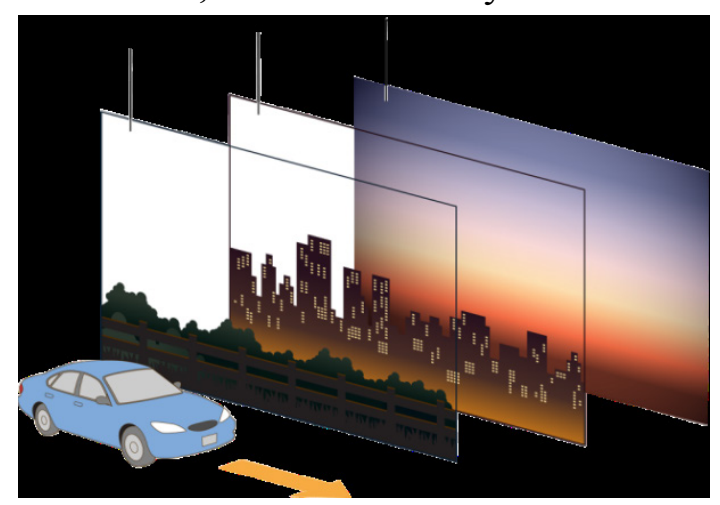

Figure 3 Multi-Layer Scrolling

will continue in 2016 .

An illustrating example of a website using the parallax scrolling effect is:

http://chandelier.elated-themes.com/parallax/

\section{Carousel Front-Page/ Video Header}

More and more web sites turn into a cinema screen. High-speed Internet connections and better integration plug-in makes it much easier to integrate video elements in web pages and to transform websites in a compelling cinematic experience. Videos are growing from small fragments to full length videos and the distributed images are very clear, highdefinition, creating a new online video experience for users. A site must alternate 3 types of visual elements: text, image and video. 2016 put so much emphasis on improving further the user experience on a 
website and, that is why the premise of promoting a brand through video presentation or video-collage of images will increase the time and pages spent by a user per session. Therefore the following criteria must be taken into account: is it an important subject which has to be highlighted, does it ensure continuity of the information in the page, gives users the answer to certain questions? - Considering these, a video is inserted with measure.

\section{Flat Design}

It is well known that a site is a harmony regarding all elements that are appearing in its development, which should have a definite purpose. Clear trend for some time now is the simplicity and modernity brought by flat design. It is based on eliminating any unnecessary elements of design and focuses on the idea of functionality. Here are some of the most important features:

- Dividing lines: flat trend in design is to remove lines and separating elements in order to give the interface a clean and functional look. For the separation of elements are increasingly used more blocks of different colors, see figure 4. The ultimate goal is to simplify the interface as much to make it more functional.

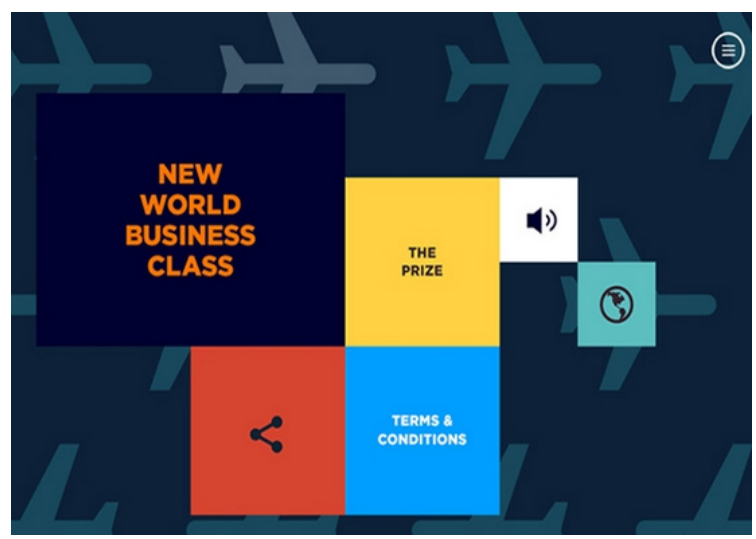

Figure 4 More blocks of different colors

- Simplifying color scheme and interface elements: using simple color schemes associated with flat design and philosophy less is more. General schemes are composed of neutral colors and buttons call to action using strong colors both for graphical impact that it creates and for causing the user to make the desired action. Simplifying the interface improves user experience while using too many colors can have a negative impact.

- Quitting decorative patterns and gradient: a few years ago most websites were characterized by gradient backgrounds (gradients) or decorative patterns. These items are completely out of the equation in flat design.

- Using icons: websites and applications use icons a lot and especially fonts shaped as icons, see figure 5. These range from general icons for web applications, to those for web forms control, from the icons for the most used currencies, to those that are characteristic of text editors, to the directional ones, or those for social networks.
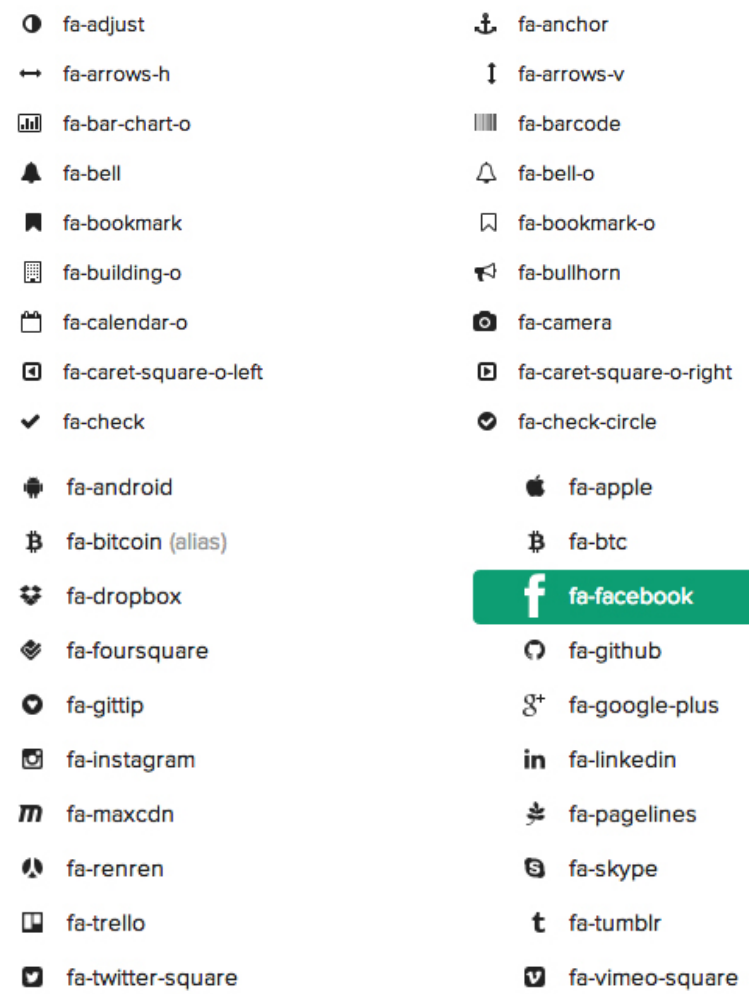

Figure 5 Web Application and Brand Icons

\section{Loading Type Animations}

Surprisingly, animations to load web pages returned in trend. They were in vogue especially in the years when flash animations were present in most websites. Due to the typically large size of SWG files created in action script, an animation was 


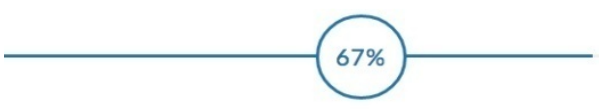

Figure 6 Loading Type Animation

needed to keep the visitor's attention until the script loaded, see figure 6 .

This return can find its explanation in the fact that the size of web pages increases from year to year. According to an article published by Adobe, web pages migrated from medium size $700 \mathrm{~Kb}$ in 2010 to $2200 \mathrm{~Kb}$ in 2015 .

\section{The Blur Technique}

Used to load images, this CSS technique consists in the initial loading of small images that blur effect has been applied, that in the end when fully charged, the image to be visible at the normal size, see figure 7 .

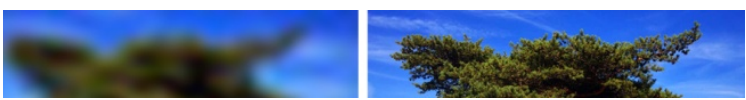

Figure 7 Applying the Blur Technique

It is useful to avoid situations that meet especially when browsing on mobile devices, where space remains empty until the full charge. It is a technique that speeds up page loading by $30 \%$.

\section{Cards Layout}

Organizing layouts in cards has become a widely used practice in web design following Pinterest social network model. If we study current websites, we notice that information is placed in the form

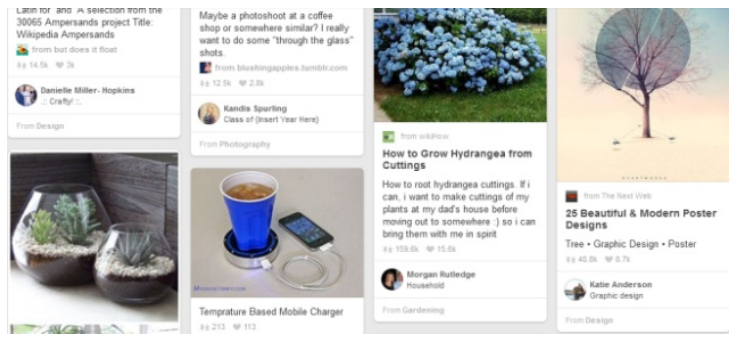

of cards. Websites like Pinterest, Google+, and Twitter use this technique for the information to be taken with ease and in a more accessible manner, see figure 8 .

Pinterest is the leader in this regard, but we see increasingly more blogs and online stores using this method for promotion.

Reasons that layouts organized in cards have success and are widely used are multiple:

- help delineate sections clearly and can accommodate a large amount of information;

- are easy to adjust items in responsive layouts;

- contribute to a clean and organized layout yet complex;

- can accommodate both images and text in multiple columns per row;

\section{Conclusions}

Looking at the trends examples above, it is easy to see that there is not only a design trend that designers will focus on this year but a combination of several trends in recent years. The online environment is constantly changing, so designing a great website can be difficult, and to be original is even more difficult. Considering this premise, it is important to be always aware of trends in terms of design. The first step to having an original site is the analysis of design, content and its functionality. An important clarification is that the trends described above are not applicable everywhere, creativity being an essential ingredient for creating each site. According to a Stanford University credibility study, over $70 \%$ of Internet users judge the credibility of a company after the site's web design. In conclusion, when it comes to our company's online presence people judge the book by its cover.

Figure 8 Cards Layout (https://ro.pinterest.com)

\section{References}

[1] UXPin, e-book Web Design Book of Trends 2015 \& 2016, Copyright (C) 2015 by UXPin Inc.

[2] http://designingfortheweb.co.uk

[3] UXPin, e-book Mobile Design Book of Trends 2015 \& 2016, Copyright (C) 2015 by UXPin Inc. 